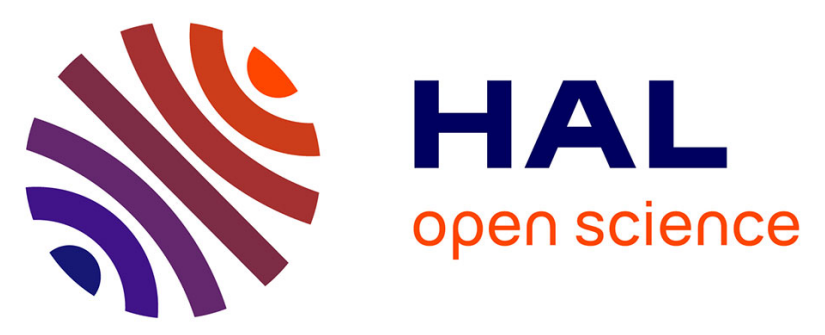

\title{
A sliding mode observer for linear systems with unknown time-varying delay
}

Alexandre Seuret, Thierry Floquet, Jean-Pierre Richard, Sarah K. Spurgeon

\section{To cite this version:}

Alexandre Seuret, Thierry Floquet, Jean-Pierre Richard, Sarah K. Spurgeon. A sliding mode observer for linear systems with unknown time-varying delay. Automatic Control Conference, Jul 2007, New York City, United States. pp.4558 - 4563, 10.1109/ACC.2007.4282449 . hal-00385904

\section{HAL Id: hal-00385904 https://hal.science/hal-00385904}

Submitted on 20 May 2009

HAL is a multi-disciplinary open access archive for the deposit and dissemination of scientific research documents, whether they are published or not. The documents may come from teaching and research institutions in France or abroad, or from public or private research centers.
L'archive ouverte pluridisciplinaire HAL, est destinée au dépôt et à la diffusion de documents scientifiques de niveau recherche, publiés ou non, émanant des établissements d'enseignement et de recherche français ou étrangers, des laboratoires publics ou privés. 


\title{
A sliding mode observer for linear systems with unknown time varying delay
}

\author{
Alexandre Seuret, Thierry Floquet, Jean-Pierre Richard and Sarah K. Spurgeon *
}

\begin{abstract}
The design of observers for linear systems with unknown, time-varying, bounded delays (on the state and on the input) still constitutes an open problem. In this paper, we show how to solve it for a class of systems by combining the sliding mode observer approach with an adequate choice of a Lyapunov-Krasovskii functional. This result provides workable conditions in terms of rank assumptions and LMI conditions. The dynamic properties of the observer are also analyzed. A 4th-order example is proposed to study the feasibility.
\end{abstract}

Sliding Mode Observer, Time-Delay Systems, Unknown Delay, Linear Matrix Inequalities.

\section{Introduction}

State observation is an important issue for both linear and nonlinear systems. This work considers the observation problem in the case of linear systems with unknown delay. Several authors proposed observers for delay systems (see, e.g., [21, 20]). Most of them, as it is pointed out in [20], consider that the value of the delay (mainly constant) can be involved in the observer realization. Concretely, this means that the delay is known or measured. Likewise, what is defined as "observers without internal delay" $[4,5,11]$ involves the output knowledge at the present and delayed instants. Besides, in [16] was designed a finite-dimensional observer (thus, without delay) since it was constructed just for the finite set of unstable or poorly damped modes of the delay system. However, the determination of these modes, here again, requires the delay knowledge.

Yet, in concrete applications (for instance teleoperation, or networked systems), the delay invariance and delay knowledge remain assumptions coming more from the

${ }^{*}$ A. Seuret, T. Floquet and J.-P. Richard are with LAGIS CNRS UMR 8146, Ecole Centrale de Lille, BP 48, 59651 Villeneuve d'Ascq Cedex, France (seuret.alexandre, thierry.floquet, jean-pierre.richard) dec-lille.fr

${ }^{\dagger}$ S. K. Spurgeon is with the Control \& Instrumentation Research Group, Department of Engineering, Leicester University, UK eoneleicester.ac.uk

†This work was also supported by an EPSRC Platform Grant reference EP/D029937/1 entitled "Control of Complex Systems" identification and analysis limits than from technical facts. There are presently only few results in which the observer does not assume the delay knowledge [2, 3, 7, 14, 26]. These interesting approaches consider linear systems and guarantee an $H_{\infty}$ performance for the filtering error. Those approaches are based on i.o.d stability techniques (independent of the delay). So it should be interesting to reduce the probable conservatism of such results by taking into account the information on a delay upper-bound.

In this paper, we propose a method to solve the problem of the observation of linear systems with unknown time delays by combining some results on sliding mode observers (see, e.g., $[1,8,9,12,19]$ ) with an adequate choice of a Lyapunov-Krasovskii functional. The observer dynamical properties will also be discussed. For the sake of simplicity, the unknown time delay $h(t)$ is assumed to be the same for the state and the input. In order to reduce the conservatism of the worked out conditions, it is supposed to have a known upper bound $h_{m}$ so that:

$$
0 \leq h(t) \leq h_{m}, \forall t \in \mathbb{R}_{+} .
$$

Throughout the article, the notation $P>0$ for $P \in \mathbb{R}^{n \times n}$ means that $P$ is a symmetric and positive definite matrix. $\left[A_{1}\left|A_{2}\right| \ldots \mid A_{n}\right]$ is the concatenated matrix with matrices $A_{i}$. $I_{n}$ represents the $n \times n$ identity matrix. Finally, $\operatorname{Sym}\{P\}=$ $\left(P+P^{T}\right)$.

\section{Problem statement}

Let us consider the linear time-invariant system with state and input delay:

$$
\left\{\begin{aligned}
\dot{x}(t)= & A x(t)+A_{h} x(t-h(t)) \\
& +B u(t)+B_{h} u(t-h(t))+D \zeta(t) \\
y(t)= & C x(t) \\
x(s)= & \phi(s), \quad \forall s \in\left[-h_{m}, 0\right]
\end{aligned}\right.
$$

where $x \in \mathbb{R}^{n}, u \in \mathbb{R}^{m}$ and $y \in \mathbb{R}^{q}$ are the state vector, the input vector and the measurement vector, respectively. $\zeta \in$ $\mathbb{R}^{r}$ is an unknown and bounded perturbation that satisfies:

$$
\|\zeta(t)\| \leq \alpha_{1}(t, y, u)
$$

where $\alpha_{1}$ is a known scalar function. $\phi \in C^{0}\left(\left[-h_{m}, 0\right], \mathbb{R}^{n}\right)$ is the vector of initial conditions. It is assumed that 
$A, A_{h}, B, B_{h}, C$ and $D$ are constant known matrices of appropriate dimensions. The following structural assumptions are required for the design of the observer:

A1. $\operatorname{rank}\left(C\left[A_{h}\left|B_{h}\right| D\right]\right)=\operatorname{rank}\left(\left[A_{h}\left|B_{h}\right| D\right]\right) \triangleq p$,

A2. $p<q \leq n$,

A3. The invariant zeros of $\left(A,\left[A_{h}\left|B_{h}\right| D\right], C\right)$ lie in $\mathbb{C}^{-}$.

Under those assumptions and using the same linear change of coordinates as in [10], Chapter 6, the system can be transformed into:

$$
\left\{\begin{aligned}
\dot{x}_{1}(t)= & A_{11} x_{1}(t)+A_{12} x_{2}(t)+B_{1} u(t) \\
\dot{x}_{2}(t)= & A_{21} x_{1}(t)+A_{22} x_{2}(t)+B_{2} u(t) \\
& +G_{1} x_{1}(t-h(t))+G_{2} x_{2}(t-h(t)) \\
& +G_{u} u(t-h(t))+D_{1} \zeta(t) \\
y(t)= & T x_{2}(t)
\end{aligned}\right.
$$

where $x_{1} \in \mathbb{R}^{n-q}, x_{2} \in \mathbb{R}^{q}$ and where $G_{1}, G_{2}, G_{u}, D_{1}$ and $A_{21}$ are defined by:

$$
\begin{gathered}
G_{1}=\left[\begin{array}{c}
0 \\
\bar{G}_{1}
\end{array}\right], G_{2}=\left[\begin{array}{c}
0 \\
\bar{G}_{2}
\end{array}\right], G_{u}=\left[\begin{array}{c}
0 \\
\bar{G}_{u}
\end{array}\right], \\
D_{1}=\left[\begin{array}{c}
0 \\
\bar{D}_{1}
\end{array}\right], A_{21}=\left[\begin{array}{c}
A_{211} \\
A_{212}
\end{array}\right]
\end{gathered}
$$

with $\bar{G}_{1} \in \mathbb{R}^{p \times(n-q)}, \bar{G}_{2} \in \mathbb{R}^{p \times q}, \bar{G}_{u} \in \mathbb{R}^{p \times m}, \bar{D}_{1} \in \mathbb{R}^{p \times r}$, $A_{211} \in \mathbb{R}^{(q-p) \times(n-q)}, A_{212} \in \mathbb{R}^{p \times(n-q)}$ and $T$ an orthogonal matrix involved in the change of coordinates given in [10].

Under conditions $A$, the system can be decomposed in two subsystems. $A 1$ implies that the unmeasurable state $x_{1}$ is not affected by the delayed terms and the perturbations. $A 3$ ensures that the pair $\left(A_{11}, A_{211}\right)$ is at least detectable.

In this article, the following lemma will be used:

Lemma 1 [15] For any matrices $A, P_{0}>0$ and $P_{1}>0$, the inequality

$$
A^{T} P_{1} A-P_{0}<0,
$$

is equivalent to the existence of a matrix $Y$ such that:

$$
\left[\begin{array}{cc}
-P_{0} & A^{T} Y^{T} \\
Y A & -Y-Y^{T}+P_{1}
\end{array}\right]<0 .
$$

\section{Observer design}

Let us define the following sliding mode observer:

$$
\left\{\begin{aligned}
\dot{\hat{x}}_{1}(t)= & A_{11} \hat{x}_{1}(t)+A_{12} x_{2}(t)+B_{1} u(t) \\
& +\left(L T^{T} G_{l} T-A_{11} L\right)\left(x_{2}(t)-\hat{x}_{2}(t)\right)+L T^{T} v(t) \\
\dot{\hat{x}}_{2}(t)= & A_{21} \hat{x}_{1}(t)+A_{22} x_{2}(t)+B_{2} u(t) \\
& -\left(A_{21} L+T^{T} G_{l} T\right)\left(x_{2}(t)-\hat{x}_{2}(t)\right) \\
& +G_{1} \hat{x}_{1}(t-\hat{h})+G_{2} x_{2}(t-\hat{h})+G_{u} u(t-\hat{h}) \\
& -G_{1} L\left(x_{2}(t-\hat{h})-\hat{x}_{2}(t-\hat{h})\right)-T^{T} v(t) \\
\hat{y}(t)= & T \hat{x}_{2}(t)
\end{aligned}\right.
$$

where the linear gain $G_{l}$ is an Hurwitz matrix and $L$ has the form $\left[\begin{array}{cc}\bar{L} & 0\end{array}\right]$ with $\bar{L} \in \mathbb{R}^{(n-q) \times(q-p)}$. The computed delay $\hat{h} \leq h_{m}$ is an implemented value that can be chosen according to the parameters of the system. It could also be time-varying. For instance $\hat{h}$ could be equal to some expected nominal estimation of the time-varying delay. The discontinuous injection term $v$ is given by:

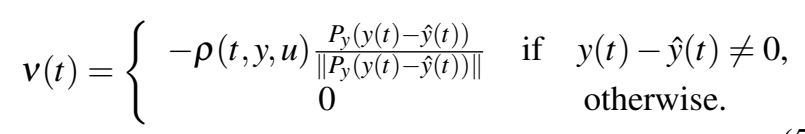

where $P_{y}>0, P_{y} \in \mathbb{R}^{p \times p}$ and where $\rho$ is a nonlinear positive gain yet to be defined. Note that the non delayed terms depending on $x_{2}$ are known because $x_{2}(t)=T^{T} y(t)$. Defining the state estimation errors as $e_{1}=x_{1}(t)-\hat{x}_{1}(t)$ and $e_{2}=x_{2}(t)-\hat{x}_{2}(t)$, one obtains:

$$
\left\{\begin{aligned}
\dot{e}_{1}(t)= & A_{11} e_{1}(t)-L\left(T^{T} G_{l} T e_{2}(t)+T^{T} v(t)\right) \\
& +A_{11} L e_{2}(t) \\
\dot{e}_{2}(t)= & A_{21} e_{1}(t)+G_{1} e_{1}(t-h(t))+D_{1} \zeta(t) \\
& +T^{T} v(t)+\xi_{0}(t)+\left(T^{T} G_{l} T+A_{21} L\right) e_{2}(t) \\
& +G_{1} L e_{2}(t-\hat{h})
\end{aligned}\right.
$$

where $\xi_{0}: \mathbb{R} \longmapsto \mathbb{R}^{p}$ is given by:

$$
\begin{aligned}
\xi_{0}(t)= & G_{1}\left(\hat{x}_{1}(t-h(t))-\hat{x}_{1}(t-\hat{h})\right) \\
& +G_{2}\left(x_{2}(t-h(t))-x_{2}(t-\hat{h})\right) \\
& +G_{u}(u(t-h(t))-u(t-\hat{h})) .
\end{aligned}
$$

Let us introduce the change of coordinates $\left[\begin{array}{l}\bar{e}_{1} \\ \bar{e}_{2}\end{array}\right]=$ $T_{L}\left[\begin{array}{l}e_{1} \\ e_{2}\end{array}\right]$ with $T_{L}=\left[\begin{array}{cc}I_{n-q} & L \\ 0 & T\end{array}\right]$. Using the fact that $L G_{1}=L G_{2}=L G_{u}=L D_{1}=0$, one obtains:

$$
\left\{\begin{aligned}
\dot{\bar{e}}_{1}(t)= & \left(A_{11}+L A_{21}\right) \bar{e}_{1}(t) \\
\dot{\bar{e}}_{2}(t)= & T A_{21} \bar{e}_{1}(t)+T G_{1} \bar{e}_{1}(t-h(t)) \\
& +G_{l} \bar{e}_{2}(t)+v(t)+T \xi(t)+T D_{1} \zeta(t)
\end{aligned}\right.
$$

with

$$
\begin{aligned}
\xi(t)= & G_{1}\left(\hat{x}_{1}(t-h(t))-\hat{x}_{1}(t-\hat{h})\right) \\
& +G_{2}\left(x_{2}(t-h(t))-x_{2}(t-\hat{h})\right) \\
& -G_{1} L\left(e_{2}(t-h(t))-e_{2}(t-\hat{h})\right) \\
& +G_{u}(u(t-h(t))-u(t-\hat{h})),
\end{aligned}
$$

that can be rewritten as:

$$
\xi(t)=\left[\begin{array}{llll}
G_{1} & G_{2} & -G_{1} L & G_{u}
\end{array}\right] \int_{t-\hat{h}}^{t-h(t)}\left[\begin{array}{c}
\dot{\hat{x}}_{1}(s) \\
\dot{x}_{2}(s) \\
\dot{e}_{2}(s) \\
\dot{u}(s)
\end{array}\right] d s
$$


The function $\xi$ only depends on the known variables $\hat{x}_{1}$, $x_{2}, e_{2}$ and $u$ and on the unknown delay $h(t)$. One can then assume that there exists a known scalar function $\alpha_{2}$ such that:

$$
\|\xi(t)\| \leq \alpha_{2}\left(t, \hat{x}_{1}, x_{2}, e_{2}, u\right) .
$$

Remark 1 Note that the nearest the available estimation $\hat{h}$ of $h(t)$, the smallest the bound $\alpha_{2}$ (indeed $\hat{h}=h(t)$ implies $\xi=0)$. This means that an available information on the delay size order allows for reducing the observer gain. Furthermore, as it will be seen hereafter, a value $h_{m}$ of the upper-bound of the admissible time delays will be available via an LMI formulation.

It is now possible to define more precisely the "discontinuous gain" $\rho$, using the same technique for the design of the sliding mode control law in [13]:

$$
\rho(t, y, u)=\left\|D_{1}\right\| \alpha_{1}(t, y, u)+\alpha_{2}\left(t, \hat{x}_{1}, x_{2}, e_{2}, u\right)+\gamma,
$$

with $\gamma$ a positive real number. Then the following result holds:

Theorem 1 Under the conditions $A$ and (9) and for any Hurwitz matrix $G_{l}$, the system (7) is asymptotically stable for all delay $h(t) \leq h_{m}$ if there exist symmetric positive definite matrices $P_{1}$ and $R_{1} \in \mathbb{R}^{(n-q) \times(n-q)}, P_{2} \in \mathbb{R}^{q \times q}$, a symmetric matrix $Z_{2} \in \mathbb{R}^{q \times q}$ and a matrix $W \in \mathbb{R}^{(n-q) \times(q-p)}$ such that the following LMI conditions are satisfied:

$$
\begin{aligned}
& {\left[\begin{array}{ccc}
\psi_{0} & A_{11}^{T} P_{1}+A_{211}^{T} W^{T} & \left(A_{21}+G_{1}\right)^{T} T^{T} P_{2} \\
& -2 P_{1}+h_{m} R_{1} & 0 \\
* & G_{l}^{T} P_{2}+P_{2} G_{l}+h_{m} Z_{2}
\end{array}\right]<0,} \\
& {\left[\begin{array}{cc}
R_{1} & \left(T G_{1}\right)^{T} P_{2} \\
P_{2} T G_{1} & Z_{2}
\end{array}\right] \geq 0,}
\end{aligned}
$$

where $\psi_{0}=A_{11}^{T} P_{1}+P_{1} A_{11}+A_{211}^{T} W^{T}+W A_{211}$.

The observer gain $\bar{L}$ is then given by $\bar{L}=P_{1}^{-1} W$.

Consider the candidate for a Lyapunov-Krasovskii functional:

$$
\begin{aligned}
V(t)= & \bar{e}_{1}^{T}(t) P_{1} \bar{e}_{1}(t)+\bar{e}_{2}^{T}(t) P_{2} \bar{e}_{2}(t) \\
& +\int_{-h_{m}}^{0} \int_{t+\theta}^{t} \dot{\bar{e}}_{1}^{T}(s) R_{1} \dot{\bar{e}}_{1}(s) d s d \theta .
\end{aligned}
$$

Using the following transformation:

$$
\bar{e}_{1}(t-h(t))=\bar{e}_{1}(t)-\int_{t-h(t)}^{t} \dot{\bar{e}}_{1}(s) d s,
$$

and differentiating (13) along the trajectories of (7), one gets:

$$
\begin{aligned}
\dot{V}(t)= & \bar{e}_{1}^{T}(t)\left[\operatorname{Sym}\left\{\left(A_{11}+L A_{21}\right)^{T} P_{1}\right\}\right] \bar{e}_{1}(t) \\
& +\bar{e}_{2}^{T}(t)\left[G_{l}^{T} P_{2}+P_{2} G_{l}\right] \bar{e}_{2}(t) \\
& +\operatorname{Sym}\left\{\bar{e}_{2}^{T}(t) P_{2} T\left(A_{21}+G_{1}\right) \bar{e}_{1}(t)\right\} \\
& -2 \rho(t, y, u)\left\|P_{2} \bar{e}_{2}(t)\right\|+h_{m} \dot{\bar{e}}_{1}^{T}(t) R_{1} \dot{\bar{e}}_{1}(t) \\
& +\eta_{1}(t)+\eta_{2}(t)-\int_{t-h_{m}}^{t} \dot{\bar{e}}_{1}^{T}(s) R_{1} \dot{\bar{e}}_{1}(s) d s,
\end{aligned}
$$

where

$$
\begin{aligned}
& \eta_{1}(t)=-2 \bar{e}_{2}^{T}(t) P_{2} T G_{1} \int_{t-h(t)}^{t} \dot{\bar{e}}_{1}(s) d s, \\
& \eta_{2}(t)=2 \bar{e}_{2}^{T}(t) P_{2} T\left[D_{1} \zeta(t)+\xi(t)\right] .
\end{aligned}
$$

The LMI condition (12) implies that for any vector $X$ :

$$
X^{T}\left[\begin{array}{cc}
R_{1} & \left(T G_{1}\right)^{T} P_{2} \\
P_{2} T G_{1} & Z_{2}
\end{array}\right] X \geq 0
$$

Developing this relation for $X=\left[\begin{array}{c}\dot{\bar{e}}_{1}(s) \\ \bar{e}_{2}(t)\end{array}\right]$, one has:

$$
-2 \bar{e}_{2}(t) P_{2} G_{1} \dot{\bar{e}}_{1}(s) \leq \bar{e}_{2}(t)^{T} Z_{2} \bar{e}_{2}(t)+\dot{\bar{e}}_{1}^{T}(s) R_{1} \dot{\bar{e}}_{1}(s) .
$$

By integrating this inequality with respect to the $s$ variable, one can upperbound $\eta_{1}(t)$ :

$$
\begin{aligned}
& \eta_{1}(t) \leq \int_{t-h(t)}^{t} \bar{e}_{2}^{T}(t) Z_{2} \bar{e}_{2}(t) d s+\int_{t-h(t)}^{t} \dot{e}_{1}^{T}(s) R_{1} \dot{\bar{e}}_{1}(s) d s, \\
& \eta_{1}(t) \leq h_{m} \bar{e}_{2}^{T}(t) Z_{2} \bar{e}_{2}(t)+\int_{t-h_{m}}^{t} \dot{\bar{e}}_{1}^{T}(s) R_{1} \dot{\bar{e}}_{1}(s) d s .
\end{aligned}
$$

Under the definition (10) of $\rho$ and since $T$ is an orthogonal matrix:

$$
\eta_{2}(t)-2 \rho(t, y, u)\left\|P_{2} \bar{e}_{2}(t)\right\| \leq-2 \gamma\left\|P_{2} \bar{e}_{2}(t)\right\| .
$$

Taking into account (16), (17) and that $\dot{\bar{e}}_{1}(t)=\left(A_{11}+\right.$ $\left.\bar{L} A_{211}\right) \bar{e}_{1}(t), \dot{V}$ can be upperbounded as follows:

$$
\begin{aligned}
\dot{V}(t) \leq & \operatorname{Sym}\left\{\bar{e}_{1}^{T}(t) P_{1}\left(A_{11}+\bar{L} A_{211}\right) \bar{e}_{1}(t)\right. \\
& \left.\left.+\bar{e}_{2}^{T}(t) P_{2}\left(A_{21}+G_{1}\right) \bar{e}_{1}(t)+\bar{e}_{2}^{T}(t) P_{2} G_{l}\right] \bar{e}_{2}(t)\right\} \\
& +h_{m} \bar{e}_{1}^{T}(t)\left(A_{11}+L A_{21}\right)^{T} R_{1}\left(A_{11}+L A_{21}\right) \bar{e}_{1}(t) \\
& +h_{m} \bar{e}_{2}^{T}(t) Z_{2} \bar{e}_{2}(t)-2 \gamma\left\|P_{2} e_{2}(t)\right\|,
\end{aligned}
$$

This can be rewritten as:

$$
\dot{V}(t) \leq\left[\begin{array}{c}
\bar{e}_{1}(t) \\
\bar{e}_{2}(t)
\end{array}\right]^{T} \Psi\left[\begin{array}{l}
\bar{e}_{1}(t) \\
\bar{e}_{2}(t)
\end{array}\right]-2 \gamma\left\|P_{2} \bar{e}_{2}(t)\right\|,
$$

with

$$
\Psi=\left[\begin{array}{cc}
\psi_{1} & \left(A_{21}+G_{1}\right)^{T} T^{T} P_{2} \\
P_{2} T\left(A_{21}+G_{1}\right) & G_{l}^{T} P_{2}+P_{2} G_{l}+h_{m} Z_{2}
\end{array}\right]
$$

and

$$
\begin{aligned}
\psi_{1}= & \left(A_{11}+\bar{L} A_{211}\right)^{T} P_{1}+P_{1}\left(A_{11}+\bar{L} A_{211}\right) \\
& +h_{m}\left(A_{11}+\bar{L} A_{211}\right)^{T} R_{1}\left(A_{11}+\bar{L} A_{211}\right)
\end{aligned}
$$

One can note that (19) is not a LMI condition since there are some nonlinear terms in the first row and the first column. Nevertheless, this problem can be transformed into an LMI condition using Lemma 1:

$$
\left[\begin{array}{ccc}
\psi_{0} & \left(A_{11}+\bar{L} A_{211}\right)^{T} Y^{T} & \left(A_{21}+G_{1}\right)^{T} T^{T} P_{2} \\
* & -Y-Y^{T}+h_{m} R_{1} & 0 \\
* & * & G_{l}^{T} P_{2}+P_{2} G_{l}+h_{m} Z_{2}
\end{array}\right]<0 .
$$

Let us set $Y=P_{1}$ and define $W=P_{1} \bar{L}$. The LMI conditions of Theorem 1 appear. Thus, if (11) and (12) are satisfied, (20) is also satisfied. This implies that the observation error is asymptotically stable. 


\section{Dynamic properties of the observer}

\subsection{Finite time convergence on the sliding mani- fold}

Corollary 1 Under the observer design of Theorem 1, an ideal sliding motion takes place on $S_{0}=\left\{\bar{e}_{2}=0\right\}$ in finite time.

Consider the Lyapunov function:

$$
V_{2}(t)=\bar{e}_{2}^{T}(t) P_{y} \bar{e}_{2}(t)
$$

Differentiating along the trajectories of (7), one obtains:

$$
\begin{aligned}
\dot{V}_{2}(t)= & \bar{e}_{2}^{T}(t)\left(G_{l}^{T} P_{y}+P_{y} G_{l}\right) \bar{e}_{2}(t)+2 \bar{e}_{2}^{T}(t) P_{y} T\left[T^{T} v\right. \\
& \left.+A_{21} \bar{e}_{1}(t)+G_{1} \bar{e}_{1}(t-h(t))+D_{1} \zeta(t)+\xi(t)\right] .
\end{aligned}
$$

Using the fact that $G_{l}$ is Hurwitz and (5), one can write the following upper bound for $\dot{V}_{2}(t)$ :

$$
\dot{V}_{2}(t) \leq 2\left\|P_{y} \bar{e}_{2}(t)\right\|\left[\left\|A_{21} \bar{e}_{1}(t)+G_{1} \bar{e}_{1}(t-h(t))\right\|-\gamma\right] .
$$

From Theorem 1, the error $e_{1}$ is asymptotically stable. Thus, there exist an instant $t_{0}$ and a positive scalar $\delta$ such that : $\forall t \geq t_{0}, \quad\left\|A_{21} \bar{e}_{1}(t)+G_{1} \bar{e}_{1}(t-h(t))\right\| \leq \gamma-\delta$

This leads to

$$
\begin{aligned}
\forall t \geq t_{0}, \quad \dot{V}_{2}(t) & \leq-2 \delta\left\|P_{y} \bar{e}_{2}(t)\right\| \\
& \leq-2 \delta \sqrt{\lambda_{\min }\left(P_{y}\right)} \sqrt{V_{2}(t)} .
\end{aligned}
$$

where $\lambda_{\min }\left(P_{y}\right)$ is the smallest eigenvalue of $P_{y}$. Integrating the last differential inequation, it follows that an ideal sliding motion takes place on $S_{0}$ in finite time.

\subsection{Exponential stability}

In this part, the observer convergence is improved by giving a criteria of exponential convergence. Exponential stability properties could be an interesting way to characterize the convergence rate of the observers. As in [18, 22], for some given rate $\alpha>0$, a system (7) is said to be $\alpha$-stable, or "exponentially stable with the rate $\alpha$ ", if there exists a scalar $\beta \geq 1$ such that the solution $e\left(t ; t_{0}, \phi\right)$ of $(7)$, with any initial function $\phi$, satisfies:

$$
\left|e\left(t, t_{0}, \phi\right)\right| \leq \beta|\phi| e^{-\alpha\left(t-t_{0}\right)} .
$$

In spite of the unknown and variable delay, the following Theorem ensures that the observer dynamics is $\alpha$-stable.

Theorem 2 Under conditions $A$ and (9), the system (7) is $\alpha$-stable for any delay $h(t) \leq h_{m}$ if there exist symmetric positive definite matrices $P_{1}, R_{1}$ and $R_{2} \in \mathbb{R}^{(n-q) \times(n-q)}$, $P_{2} \in \mathbb{R}^{q \times q}$, a symmetric matrix $Z_{2} \in \mathbb{R}^{q \times q}$ and a matrix
$W \in \mathbb{R}^{(n-q) \times(q-p)}$ such that the following LMI conditions are satisfied:

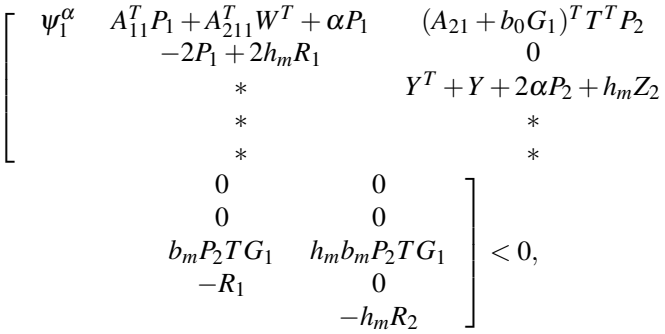

$$
\begin{aligned}
& {\left[\begin{array}{cc}
R_{1} & b_{0}\left(T G_{1}\right)^{T} P_{2} \\
b_{0} P_{2} T G_{1} & Z_{2}
\end{array}\right] \geq 0 .}
\end{aligned}
$$

where

$$
\begin{aligned}
\psi_{1}^{\alpha}= & A_{11}^{T} P_{1}+P_{1} A_{11}+2 \alpha P_{1} \\
& +A_{211}^{T} W^{T}+W A_{211}+R_{2} \\
b_{0}= & \left(1+e^{\alpha h_{m}}\right) / 2, \quad b_{m}=\left(-1+e^{\alpha h_{m}}\right) / 2
\end{aligned}
$$

The observer gains are given by $\bar{L}=P_{1}^{-1} W$ et $G_{l}=$ $P_{2}^{-1} Y$.

Let us introduce the new variable $\bar{e}_{i}^{\alpha}(t)=e^{\alpha t} \bar{e}_{i}(t)$ in(7). Then, the asymptotic convergence of $\bar{e}^{\alpha}$ implies that $\bar{e}$ is $\alpha$-stable. Equation (7) becomes:

$$
\left\{\begin{aligned}
\dot{\bar{e}}_{1}^{\alpha}(t)= & \left(A_{11}+L A_{21}+\alpha I_{n-p}\right) \bar{e}_{1}^{\alpha}(t) \\
\dot{\bar{e}}_{2}^{\alpha}(t)= & T A_{21} \bar{e}_{1}^{\alpha}(t)+\left(v+T \xi(t)+T D_{1} \zeta(t)\right) e^{\alpha t} \\
& +e^{\alpha h(t)} T G_{1} \bar{e}_{1}^{\alpha}(t-h(t))+\left(G_{l}+\alpha I_{p}\right) \bar{e}_{2}^{\alpha}(t)
\end{aligned}\right.
$$

Note that $e^{\alpha h(t)}=b_{0}+\Delta(t) b_{m}$, where $\Delta(t)$ is an unknown scalar function satisfying $\|\Delta(t)\| \leq 1$. Consider the following candidate for a Lyapunov-Krasovskii functional:

$$
\begin{aligned}
V^{\alpha}(t)= & \bar{e}_{1}^{\alpha T}(t) P_{1} \bar{e}_{1}^{\alpha}(t)+\bar{e}_{2}^{\alpha T}(t) P_{2} \bar{e}_{2}^{\alpha}(t) \\
& +2 \int_{-h_{m}}^{0} \int_{t+\theta}^{t} \dot{\bar{e}}_{1}^{\alpha T}(s) R_{1} \dot{\bar{e}}_{1}^{\alpha}(s) d s d \theta .
\end{aligned}
$$

Differentiating (26) along (25), one gets:

$$
\begin{aligned}
\dot{V}^{\alpha}(t)= & \bar{e}_{1}^{\alpha T}(t)\left[\operatorname{Sym}\left\{P_{1}\left(A_{11}+\bar{L} A_{211}+\alpha I_{n-p}\right)\right\}\right] \bar{e}_{1}^{\alpha}(t) \\
& +2 \bar{e}_{2}^{\alpha T}(t) P_{2} T\left(A_{21}+b_{0} G_{1}\right) \bar{e}_{2}^{\alpha}(t) \\
& +\eta_{1}^{\alpha}(t)+\eta_{2}^{\alpha}(t)+\eta_{3}^{\alpha}(t)+\eta_{4}^{\alpha}(t) \\
& +\bar{e}_{2}^{\alpha T}(t)\left[P_{2}\left(G_{l}+\alpha I_{p}\right)+\left(G_{l}+\alpha I_{p}\right)^{T} P_{2}\right] \bar{e}_{2}^{\alpha}(t) \\
& +2 h_{m} \dot{e}_{1}^{\alpha T} R_{1} \dot{e}_{1}^{\alpha}(t)-2 \int_{t-h(t)}^{t} \dot{e}_{1}^{\alpha T}(s) R_{1} \dot{e}_{1}^{\alpha}(s) d s,
\end{aligned}
$$

$$
\begin{aligned}
& \eta_{1}^{\alpha}(t)=2 \bar{e}_{2}^{\alpha T}(t) P_{2} b_{0} T G_{1} \int_{t-h(t)}^{t} \dot{e}_{1}^{\alpha}(s) d s, \\
& \eta_{2}^{\alpha}(t)=2 \bar{e}_{2}^{\alpha T}(t) P_{2} b_{m} \Delta(t) T G_{1} e_{1}^{\alpha}(t), \\
& \eta_{3}^{\alpha}(t)=2 \bar{e}_{2}^{\alpha T}(t) P_{2} b_{m} \Delta(t) T G_{1} \int_{t-h(t)}^{t} \dot{e}_{1}^{\alpha}(s) d s, \\
& \eta_{4}^{\alpha}(t)=2 \bar{e}_{2}^{\alpha T}(t) P_{2}\left(v+T \xi(t)+T D_{1} \zeta(t)\right) e^{\alpha t} .
\end{aligned}
$$

Following the lines of Theorem 1, (24) gives a majoration of $\eta_{1}$ :

$$
\eta_{1}^{\alpha}(t) \leq h_{m} \bar{e}_{2}^{\alpha T}(t) Z_{2} \bar{e}_{2}^{\alpha}(t)+\int_{t-h_{m}}^{t} \dot{\bar{e}}_{1}^{\alpha T}(s) R_{1} \dot{\bar{e}}_{1}^{\alpha}(s) d s .
$$


For any $n \times n$ matrix $R>0$ and for any vectors $a \in R^{n}$ and $b \in R^{n}$ :

$$
\pm 2 a^{T} b \leq a^{T} R^{-1} a+b^{T} R b
$$

Let us apply (29) for $\eta_{2}^{\alpha}(t)$ with:

$$
\begin{aligned}
a^{T} & =\bar{e}_{2}^{\alpha T}(t) P_{2} b_{m} T G_{1} \Delta(t) \\
b & =e_{1}^{\alpha}(t) \\
R & =R_{2}
\end{aligned}
$$

One gets:

$$
\begin{aligned}
\eta_{2}^{\alpha}(t) \leq & \bar{e}_{2}^{\alpha T}(t) b_{m} P_{2} T G_{1} R_{2}^{-1} b_{m}\left(T G_{1}\right)^{T} P_{2}^{T} \bar{e}_{2}^{\alpha}(t) \\
& +\bar{e}_{1}^{\alpha T}(t) R_{2} \bar{e}_{1}^{\alpha}(t)
\end{aligned}
$$

Using again (29) for $\eta_{3}^{\alpha}(t)$ with:

$$
\begin{aligned}
a^{T} & =\bar{e}_{2}^{\alpha T}(t) P_{2} b_{m} T G_{1} \Delta(t) \\
b & =\dot{e}_{1}^{\alpha}(s) \\
R & =R_{1}
\end{aligned}
$$

one has:

$$
\begin{aligned}
\eta_{3}^{\alpha}(t) \leq & h_{m} \bar{e}_{2}^{\alpha T}(t) b_{m} P_{2} T G_{1} R_{1}^{-1} b_{m}\left(T G_{1}\right)^{T} P_{2}^{T} \bar{e}_{2}^{\alpha}(t) \\
& +\int_{t-h_{m}}^{t} \bar{e}_{1}^{\alpha T}(s) R_{1} \bar{e}_{1}^{\alpha}(s) d s
\end{aligned}
$$

With the discontinuous output injection $v$ defined in (5) and (10), one has:

$$
\eta_{4}^{\alpha}(t) \leq-2 \gamma\left\|P_{2} \bar{e}_{2}(t)\right\|
$$

Then, combining (28-32) with (27) leads to:

$$
\dot{V}^{\alpha}(t) \leq\left[\begin{array}{c}
\bar{e}_{1}(t) \\
\bar{e}_{2}(t)
\end{array}\right]^{T} \Psi^{\alpha}\left[\begin{array}{l}
\bar{e}_{1}(t) \\
\bar{e}_{2}(t)
\end{array}\right]-2 \gamma\left\|P_{2} \bar{e}_{2}(t)\right\|,
$$

with:

$$
\begin{array}{ll} 
& \Psi^{\alpha}=\left[\begin{array}{cc}
\psi_{11}^{\alpha} & \left(A_{21}+G_{1}\right)^{T} T^{T} P_{2} \\
* & \psi_{22}^{\alpha}
\end{array}\right] \\
\psi_{11}^{\alpha}= & \operatorname{Sym}\left\{P_{1}\left(A_{11}+\bar{L} A_{211}+\alpha I_{n-p}\right)\right\}+\left(A_{11}\right. \\
& \left.+\bar{L} A_{211}+\alpha I_{n-p}\right)^{T} R_{1}\left(A_{11}+\bar{L} A_{211}+\alpha I_{n-p}\right), \\
\psi_{22}^{\alpha}= & G_{l}^{T} P_{2}+P_{2} G_{l}+2 \alpha P_{2}+h_{m} Z_{2} \\
& +h_{m} b_{m} P_{2} T G_{1} R_{2}^{-1}\left(T G_{1}\right)^{T} P_{2} b_{m} \\
& +b_{m} P_{2} T G_{1} R_{1}^{-1}\left(T G_{1}\right)^{T} P_{2} b_{m} .
\end{array}
$$

Again, applying Lemma 1 as in Theorem 1 yields:

$$
\left[\begin{array}{ccc}
\psi_{1}^{\alpha} & A_{11}^{T} P_{1}+A_{211}^{T} W^{T}+\alpha P_{1} & \left(A_{21}+G_{1}\right)^{T} T^{T} P_{2} \\
-2 P_{1}+2 h_{m} R_{1} & 0 \\
* & \psi_{22}^{\alpha}
\end{array}\right]<0
$$

Set $Y=P_{2} G_{l}$. Then using the Schur complement, one finds the LMI condition (23). Thus, if (23) and (24) are satisfied, the time derivative of (26) is negative definite.

\subsection{Optimization Problem}

This paragraph focusses on the optimization of the exponential decay rate $\alpha$. The greater $\alpha$ is, faster the error dynamics converge to the solution $e(t)=0$. The optimization consists in finding the greatest $\alpha$ (guaranteed speed performance of the application) such that the closed-loop system is $\alpha$-stable. This corresponds to the problem

$$
\max \alpha
$$

subject to (23) and (24) for a given $h_{m}$.

Because $\alpha$ does not appear in a linear form in (23) and (24), this problem is solved by iteratively increasing $\alpha$ until the LMI conditions become unfeasible.

\section{Example}

Consider the system with time-varying delay (3) with:

$$
\begin{gathered}
A_{11}=\left[\begin{array}{cc}
0 & 0 \\
0 & -1
\end{array}\right], A_{12}=\left[\begin{array}{cc}
-1 & 0 \\
0 & 0.1
\end{array}\right], \\
A_{21}=\left[\begin{array}{cc}
2 & 3 \\
2 & -1
\end{array}\right], A_{22}=\left[\begin{array}{cc}
-1 & 0 \\
0 & -1
\end{array}\right], \\
G_{1}=\left[\begin{array}{cc}
0 & 0 \\
0.1 & 0.21
\end{array}\right], G_{2}=\left[\begin{array}{cc}
0 & 0 \\
0.2 & 1
\end{array}\right], \\
T=\left[\begin{array}{ll}
1 & 0 \\
0 & 1
\end{array}\right], G_{u}=\left[\begin{array}{l}
0 \\
1
\end{array}\right], D_{1}=B_{1}=B_{2}=\left[\begin{array}{l}
0 \\
0
\end{array}\right],
\end{gathered}
$$

The delay is chosen as $h(t)=\frac{h_{m}}{2}\left(1+\sin \left(\omega_{1} t\right)\right)$, with $h_{m}=$ $0.3 s$ an frequency $\omega_{1}=0.5 s^{-1}$. The control law is

$$
u(t)=u_{0} \sin \left(\omega_{2} t\right)
$$

with $u_{0}=2$ and $\omega_{2}=3$.

Since the system (3) is open loop stable, its dynamics are bounded. Thus the function $\alpha_{2}\left(t, \hat{x}_{1}, x_{2}, e_{2}, u\right)$ could be chosen as a constant $K=0,7$.

The simulation results are given in the following figures. In Figures 1 and 2 are reported the observation errors of the system for $\alpha=0$ and $\alpha=2$.

Figures 3 and 4 show the comparison between the real and observed states, for $\alpha=2$.

It can be noticed that the greater $\alpha$ is, the faster the error convergence is. Using Theorem 2, the following observer gains for $\alpha=2$ are obtained:

$$
\bar{L}=\left[\begin{array}{c}
-3.8658 \\
1.0722
\end{array}\right], G_{l}=\left[\begin{array}{cc}
-8.8160 & -6.0190 \\
-5.8154 & -32.0670
\end{array}\right]
$$

\section{Conclusion}

The problem of designing observers for linear systems with unknown variable delay on both input and state has 


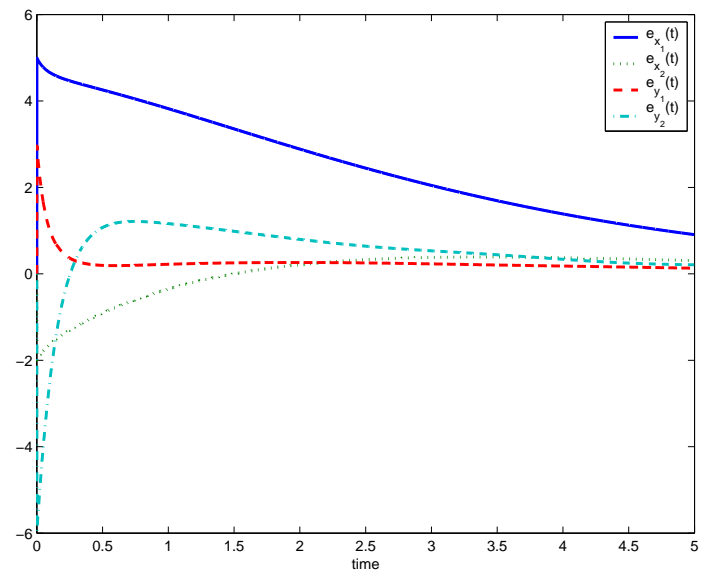

Figure 1. Observation errors for $\alpha=0$ and $h_{m}=0.3$

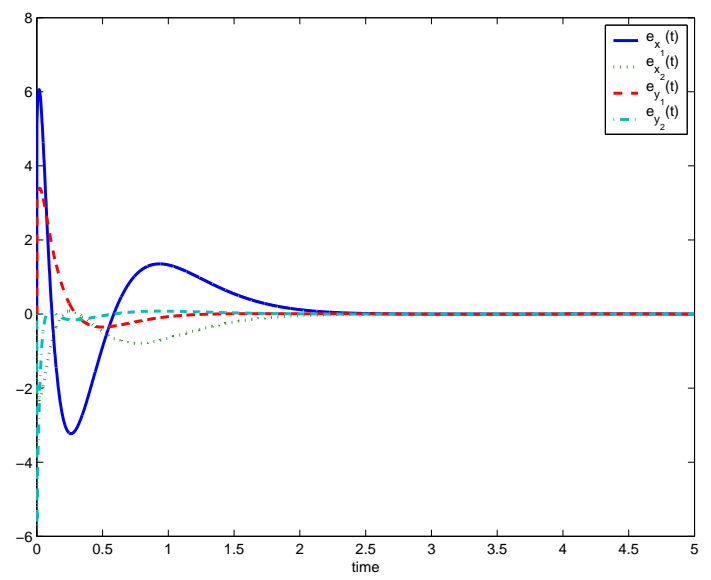

Figure 2. Observation errors for $\alpha=2$ and $h_{m}=0.3$

been solved in this article. Delay-dependent LMI conditions have been found to guarantee asymptotic stability of the dynamical error system. In addition, the dynamics properties of the proposed observer can be characterized through finite time and exponential convergence properties.

\section{References}

[1] J.-P. Barbot, T. Boukhobza and M. Djemaï, "Sliding mode observer for triangular input form". 35-th IEEE CDC'99, Conference on Decision and Control, Kobe, Japan, 1996.

[2] H. H. Choi, M. J. Chung, "Observer-based $H_{\infty}$ controller design for state delayed linear systems". Automatica, 32(7), pp. 1073-1075, 1996.

[3] H. H. Choi and M. J. Chung, "Robust Observer-based $H_{\infty}$ controller design for linear uncertain time-delay systems". Automatica, 33(9), pp. 1749-1752, 1997.

[4] M. Darouach, "Linear functional observers for systems with delays in the state variables". IEEE trans. on Automatic

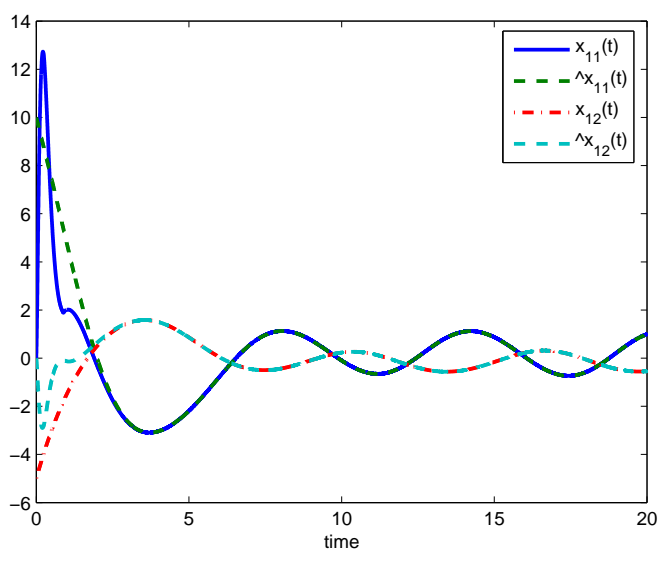

Figure 3. $x_{1}$ and $\hat{x}_{1}$, for $\alpha=2$,

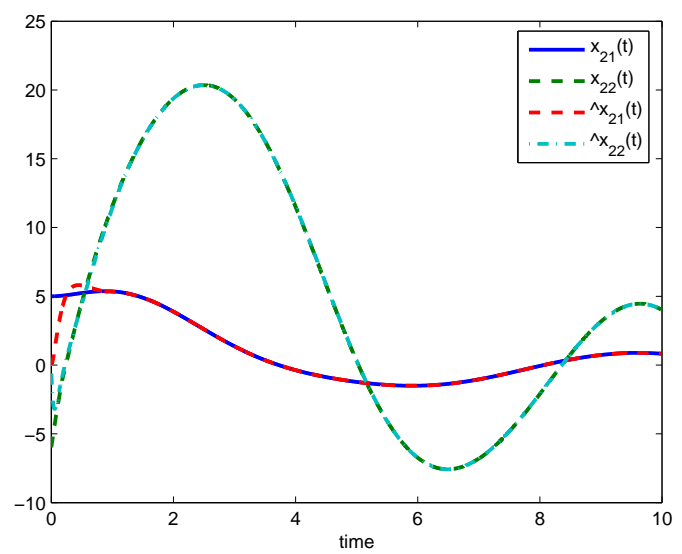

Figure 4. $x_{2}$ and $\hat{x}_{2}$, for $\alpha=2$

Control, 46(3), pp. 491-497, March 2001.

[5] M. Darouach, P. Pierrot and E. Richard, "Design of reducedorder observers without internal delays". IEEE Trans. on Automatic Control, 44(9), pp. 1711-1713, 1999.

[6] M. Darouach, M. Zasadzinski and S. J. Xu, "Full-order observers for linear systems with unknown inputs". IEEE Trans. on Automatic Control, vol. 39, pp. 606-609, 1994.

[7] C.E. de Souza, R.E. Palhares and P.L.D. Peres, "Robust $H_{\infty}$ filtering for uncertain linear systems with multiple timevarying state: An LMI approach". 38-th IEEE CDC'99, Conference on Decision and Control, Phoenix, AZ, pp. 2023-2028, 1999.

[8] S. V. Drakunov, "Sliding-mode Observer Based on Equivalent Control Method". 31st IEEE CDC'92, Conference on Decision and Control, Tucson, Arizona, 1992.

[9] C. Edwards and S. K. Spurgeon, "On the development of discontinuous observers". International Journal of Control, 59, pp. 1211-1229, 1994.

[10] Edwards C. and Spurgeon S. K., Sliding Mode Control: The- 
ory and Applications, Taylor \& Francis, 1998.

pp. 231-236, 1999.

[11] F. W. Fairmar, Kumar and E. Richard, "Design of reducedorder observers without internal delays". IEEE trans. on Automatic Control, 44(9), pp. 1711-1713, 1999.

[12] T. Floquet, J.-P. Barbot, W. Perruquetti and M. Djemaï, “On the robust fault detection via a sliding mode disturbance observer". International Journal of control, 77(7), pp. 622-629, 2004.

[13] E. Fridman, F. Gouaisbaut, M. Dambrine and J.-P. Richard, "Sliding mode control of systems with time-varying delays via a descriptor approach”. Int. J. System Sc. Vol. 34, No.89, pp. 553-559, July 2003.

[14] E. Fridman, U. Shaked and L. Xie, "Robust $H_{\infty}$ Filtering of linear systems with time-varying delay". IEEE Trans. on Automatic Control, vol. 48, pp. 159-165, 2003.

[15] L.-S. Hu, J. Huang and H.-H. Cao, "Robust digital model predicitve control for linear uncertain systems with saturations". IEEE Trans. on Automatic Control, vol. 49(5), pp. 792-796, 2004.

[16] J. Leyva-Ramos and A. E. Pearson, "An asymptotic modal observer for linear autonomous time lag systems". IEEE Trans. on Automatic Control, 40, 1291-1294, 1995.

[17] S.I. Niculescu, "Delay effects on stability", LNCIS series, Vol. 269, Springer, Berlin, 2001.

[18] S-I. Niculescu, C-E. de Souza, L. Dugard and J-M. Dion, "Robust exponential Stability of uncertain systems with time-varying delays", IEEE Trans. on Automatic Control, Vol. 43(5), pp. 743-748, 1998.

[19] W. Perruquetti and J. P. Barbot, Sliding Mode Control in Engineering, Ed. Marcel Dekker, 2002.

[20] J.-P. Richard, "Time Delay Systems: An overview of some recent advances and open problems". Automatica 39(10), pp. 1667-1694, 2003.

[21] O. Sename, "New trends in design of observers for timedelay systems". Kybernetica, 37(4), pp. 427-458, 2001.

[22] A. Seuret, M. Dambrine and J.P. Richard, "Robust exponential stabilization for systems with time-varying delays", 5-th IFAC Workshop on Time-Delay Systems, Leuven 2004.

[23] C. P. Tan, and C. Edwards, "An LMI approach for designing sliding mode observers". International Journal of Control, 74(16), pp. 1559-1568, 2001.

[24] Utkin, V. I. Sliding Modes in Control and Optimization, Berlin, Germany, Springer-Verlag, 1992.

[25] S. Xu, P. Shi, C. Feng, Y. Guo and Y. Zou, "Robust Observers for a class of uncertain nonlinear stochastic systems with state delay". Nonlinear Dynamics and systems Theory, 4(3), 2004.

[26] Z. Wang, B. Huang and H. Unbehausen, "Robust $H_{\infty}$ observer design for uncertain time-delay systems:(I) the continuous case". IFAC 14-th world congress, Beijing, China, 